\title{
Neuroprotective potential of Naringenin-loaded solid-lipid nanoparticles against rotenone-induced Parkinson's disease model
}

\author{
Megala Mani, Shivaramakrishnan Balasubramanian*, Kishore R. Manikandan, Balashanmugam Kulandaivel \\ Department of Pharmacology, JSS College of Pharmacy, JSS Academy of Higher Education \& Research, Ooty, Nilgiris, Tamil Nadu, India.
}

\begin{tabular}{l}
\hline ARTICLE INFO \\
\hline Received on: $21 / 07 / 2020$ \\
Accepted on: 03/12/2020 \\
Available online: 05/02/2021 \\
\hline Key words: \\
Parkinson's disease, \\
oxidative stress, antioxidants, \\
Naringenin, solid-lipid \\
nanoparticles, rotenone.
\end{tabular}

\section{INTRODUCTION}

Neurodegenerative disorders are the second most leading cause of death after cardiovascular diseases in humans. As per the World Health Organization, publications made on neurological disorders lists Parkinson's disease (PD) as one of the neurodegenerative disorders with reported deaths of $0.18 \%$ of the world population in the year 2005 and projected a score of $0.23 \%$ by the year 2030 (WHO, 2020). Incidentally, in the global scenario, from the year 1990 to 2016, prevalence rates of PD have more than doubled, making it the largest increase worldwide (Dorsey et al., 2018). The important point of concern is that the mortality rate is still a criteria considered to determine the severity of a disease; however, the disability rate was not (Goetz, 2011). No precise demographic model is available to predict the rate of

\section{"Corresponding Author}

Shivaramakrishnan Balasubramanian, Department of Pharmacology, JSS College of Pharmacy, JSS Academy of Higher Education \& Research, Ooty, India.E-mail: shivaram.krishna@jssuni.edu.in incidences and an alarming number of increasing deaths over the decades pose a tough task for global healthcare for PD.

Currently, the therapy involves the use of classes of drugs that either enhance the dopamine levels in the brain or mimic the effect of dopamine. The challenges for the healthcare system include the complexity of therapeutic targets to treat the disease and the need for a multi-disciplinary treatment approach for disease management (Vos et al., 2015). The current areas of research in PD are primarily focused on elucidating the molecular pathway of disease progression and early diagnostic markers (Hauser, 2009). Secondly, research on pharmacological therapy and non-pharmacological methods, namely surgery, gene therapy, and stem cell therapy, are taken up by researchers and clinicians alike (Jankovic \& Poewe, 2012). Lastly, the focus has been on the availability of uniform healthcare systems and trained personnel in low-income and developing countries like India in treating PD (Tsouli \& Konitsiotis, 2010).

Among the multiple pathways that can cause PD pathogenesis, oxidative stress is among them (Hwang, 2013). Free radicals generated in the body react with membrane lipids leading to lipid peroxidation (LPO), membrane injury, and nerve 
cell death. Research shows that the substantia nigra of patients diagnosed with PD was found to have an increase in iron level and decrease in tissue glutathione level. In addition, the lack of ability of neurons to regenerate and the outcome of free radical-induced cell injury may ultimately contribute to dopaminergic neuronal death (Collins, 2012; Sita et al., 2016).

Over the years, the consumption of antioxidants has shown to have beneficial effects in the prevention of neurodegeneration. Recently, well-balanced diets rich in natural antioxidants are of importance due to their ability to provide a variety of chemical molecules that activate or enhance the action of several endogenous antioxidants (Sun et al., 2008). Consumption of these antioxidant substances can improve motor and cognitive skills over a period of time (Joseph et al., 1999). Extensive researches have proven that antioxidants are efficient organ-protective agents against oxidative stress. However, crossing of antioxidants across blood-brain barrier (BBB) was a hindrance. Therefore, different structural antioxidants, namely stilbenes, flavanols, flavonols, isoflavones, and flavanones, were screened by Accelrys Discovery Studio v4.1 (San Diego, CA) to select a molecule that may have the potential to cross $\mathrm{BBB}$ and exerts its free radical scavenging activity against oxidative stress in PD (Ponnan et al., 2013; Vauzour, 2012).

Naringenin, a metabolic product of Naringin, was identified, screened, and studied for its potential neuroprotective property (Kumar et al., 2010). It is known to exhibit an antioxidant effect in different target tissues, scavenge free radicals, provide anti-inflammatory effect, promote carbohydrate metabolism, and lastly modulate the immune system (Chen et al., 2019; Raza et al., 2013). Studies have reported that the antioxidant characteristics of Naringenin are due to the presence of phenolic rings that act as an electron trap. It scavenges peroxy radical, superoxide anion, and prevents the oxidation of iron. Naringenin has three phenolic groups and a keto group which can react with hydroxyl radical and hydrogen peroxide, thereby inhibiting oxidative reactions (Apostolova \& Victor, 2015; Cui et al., 2014). Rotenone (ROT) is one of the common and commercially available pesticides that are reported to inhibit mitochondrial complex-I activity. By this virtue, ROT is capable of inducing mitochondrial dysfunction as seen with PD in experimental pharmacology (Manjunath \& Muralidhara, 2015). When administered aptly, the resulting effect of ROT causes oxidative stress by mitochondrial dysfunction and produces degeneration of neurons.

Solid-lipid nanoparticle (SLN) delivery system is found to be more advantageous over conventional delivery system for delivering drug into the brain (Gastaldi et al., 2014). This helps in resolving any issues with the solubility, permeability, and toxicity of a drug (Bilia et al., 2014; Kaur et al., 2008). This formulation would increase the penetration of Naringenin into the brain. With an increase in bioavailability in brain tissues, a better therapeutic effect of neuroprotection in PD may be possible. This study comprises in silico pharmacokinetic profiling of antioxidants, formulation of Naringenin into SLNs, and evaluation of prepared SLNs, followed by the evaluation of neuroprotective effect of Naringenin-loaded SLNs against ROT-induced PD model by using Drosophila and rodents as the animal model.

\section{MATERIALS AND METHODS}

\section{Drugs and chemicals}

Levodopa has been used as the standard drug, Naringenin as the test drug; ROT and Tween 80, all of which were purchased from Sigma Aldrich, India. Glycerol monostearate (GMS) was acquired from Kemphasol, India. F68 non-ionic surfactant (1.25 $w / v \%$ ) was acquired from Thermo Fisher Scientific, India.

\section{In silico pharmacokinetic studies}

To obtain an insight into pharmacokinetics, absorption, distribution, metabolism, and excretion, as well as the toxicity (ADMET) of the shortlisted antioxidants, DS software was used to evaluate them. The molecules epicatechin (B), genistein $(\mathrm{C})$, hesperetin $(D)$, naringenin $(E)$, quercetin $(F)$, resveratrol $(G)$, and taxifolin $(\mathrm{H})$ were analyzed in comparison with the reference molecule diazepam (A). The 2D mol file of shortlisted molecules was downloaded from Pubchem, NCBI, USA (Kim et al., 2019). The physiochemical characteristics and pharmacokinetics of phytoconstituents obtained under Lipinski's rule and ADMET descriptors from using the software were tabulated (Vauzour, 2012). The best suited candidate was chosen in comparison with the reference compound by its penetration across the blood-brain barrier (BBB) values. Furthermore, the ADMET_Polar Surface Area (PSA)_2D versus ADMET_AlogP98 plane plot suggested the best possible candidate for the study.

\section{Experimental animals}

Adult fruit flies of wild type obtained from Dept. of Studies in Zoology, University of Mysore, were 3 days old and were maintained in glass bottles in Biological Oxygen Demand (BOD) at a temperature of $22^{\circ} \mathrm{C} \pm 1^{\circ} \mathrm{C}$ and $80 \%$ relative humidity and fed with a standard diet (cornflour-based). Male fly larvae were isolated in the second instar larvae stage and the hatched adult flies were chosen for the experimentation (Manjunath \& Muralidhara, 2015).

Wistar albino rats of body weight 185-220 g were obtained from Central Animal House facility, JSSCP, Ooty. The animals were housed separately in standard cages (groups of six in a cage). They were allowed to adapt the current day-night phase, with food and water ad libitum for a period of 10 days. Animals exhibiting good movements, good consumption of diet, and normal body weight was selected for the model. The study was approved by Institutional IAEC (JSSCP/OT/M.Pharm/16/2018-19).

\section{ROT-induced PD model in Drosophila melanogaster}

Drosophila melanogaster species is a quick model to screen potential drugs for treatment against ROT-induced PD. The disease induction is quickly achievable compared to the rodent model. From the in silico data, we chose Naringenin as the potential candidate. The efficacy of Naringenin was tested by using the Drosophila model.

The flies $(n=50)$ were divided into four groups - Group 1 received vehicle treatment of cane sugar 7\% $v / v$, Group 2 was disease control which received treatment of ROT $(500 \mu \mathrm{M})$, Group 3 received treatment of Naringenin $750 \mu \mathrm{M}$ for 7 days, and Group 4 received pretreatment with Naringenin $750 \mu \mathrm{M}$ for 7 days, followed by disease induction using ROT. On day 8 , the disease induction was checked by negative geotaxis and flies were 
observed till day 15. Filter paper was used to soak the vehicle of cane sugar solution at $7 \% v / v$, as well as the drug solution. The negative geotaxis assay protocol followed in this study was described by Manjunath and Muralidhara (2015).

\section{Evaluation of formulated Naringenin-loaded solid-lipid nanoparticles (SLN)}

Numerous studies have reported that SLNs are an easy method to improve the pharmacokinetic profile of test drugs for better distribution in brain. Hence, Naringenin was formulated into SLNs by using GMS, Tween 80 (1.25 $w / v \%)$, and F68 (1.25 w/v \%) as per the protocol described by Wu et al. (2016). Furthermore, the formulated Naringenin-loaded SLNs were subjected to the following evaluation parameters. The morphology of Naringenin loaded SLNs was obtained using scanning electron microscopy (SEM). Particle size, polydispersity index (PDI), and zeta potential were determined by photon correlation spectroscopy using a Malvern Zetasizer Nano ZS90 (Malvern Instruments, Worcestershire, UK). The procedure was executed at $25^{\circ} \mathrm{C}$ with suitable dispersion preparation medium. Entrapment efficiency of Naringenin-loaded SLNs was obtained by High Performance Liquid Chromatography (HPLC) method at wavelength of $288 \mathrm{~nm}$ and calculated using the drug entrapment efficiency equation of analyzed weight of drug in SLNs with theoretical weight of drug-loaded SLNs.

\section{In vitro release studies and drug-release kinetics of Naringenin loaded SLNs}

The dialysis bag technique was used to carry out the in vitro release studies. The procedure was carried out using a dialysis bag (25 mm, Molecular Weight cutoff 12,000 Da) obtained from Sigma Aldrich, India. The procedure described by Wu et al. (2016) was used to evaluate the drug release. Phosphate-buffered saline of $0.1 \mathrm{mM}$ concentration and $\mathrm{pH} 7.4$ was used in the study to mimic blood. Naringenin-loaded SLNs suspension was loaded in dialysis bag and placed in preheated release medium $(50 \mathrm{ml})$ at $37^{\circ} \mathrm{C}$ and 100 rpm speed. Aliquots of $0.5 \mathrm{ml}$ were withdrawn and a concentration of Naringenin was analyzed by the HPLC method at a wavelength of $288 \mathrm{~nm}$. Fresh release medium was added to maintain sink conditions. In accordance with in silico studies, the data were interpreted for release kinetics at graphical interface using the Higuchi model and the regression coefficient $\left(R^{2}\right)$ was calculated.

\section{ROT-induced PD model in Wistar rats}

All the doses were administered with respect to $\mathrm{mg}$ per $\mathrm{kg}$ body weight. Each study group consisted of six animals each. Group 1 received treatment with vehicle; Group 2 received ROT as disease control, Group 3 received standard treatment of Levodopa at $25 \mathrm{mg} /$ $\mathrm{kg}$, Group 4 received treatment with Naringenin in its pure form at $50 \mathrm{mg} / \mathrm{kg}$, and Group 5 received treatment in SLN formulation of Naringenin as SLNs $50 \mathrm{mg} / \mathrm{kg}$ equivalent dose in formulation. For stereotaxic infusion of ROT, the animals were anesthetized with ketamine $100 \mathrm{mg} / \mathrm{kg}$ b.w by i.p. and xylazine $15 \mathrm{mg} / \mathrm{kg}$ b.w by i.m. and secured on a bed over the Rat Stereotaxic apparatus (Stoelting Co. Wood Dale, IL). ROT was infused into the right substantia nigra pars compacta (Anteroposterior: $5.0 \mathrm{~mm}$, Lateral: $2.0 \mathrm{~mm}$; Dorsoventral: $8.0 \mathrm{~mm}$ ). Following 5 minutes retention, adequate postoperative care was given until complete recovery (Xiong et al., 2009). As a postoperative care need, the treated animals were administered ibuprofen and penicillin.
The behavioral study of muscle grip strength was conducted to evaluate the motor coordination due to the drug treatment using rotarod apparatus to analyze the muscle grip strength. Rota rod apparatus was set at $20 \mathrm{rpm}$. Each rat was trained properly for the minimum of five trials before the actual study. The "fall of time" was noted and compared with treatment group (Campos et al., 2013; Zafar et al., 2003).

\section{Biochemical evaluation of brain homogenates}

After carbon dioxide euthanasia of the flies and rats, the brain homogenates of the whole head in case of fruit flies and rat brain were subjected to LPO test (Ohkawa et al., 1979), reduced glutathione (GSH) estimation by Ellman's (1959) method, superoxide dismutase (SOD) assay (Saggu et al., 1989), and catalase (CAT) assay by Mukherjee et al. (2007). The total brain protein estimation was estimated by Biuret's method (Wokes \& Still, 1942).

\section{Statistical analysis}

All the numerical values are expressed as Mean \pm SE. Data were analyzed by one-way analysis of variance, followed by Dunnet's post-hoc test. Statistical analysis was carried using Graphpad prism version 6.01 (San Diego, CA). Values with $p<$ 0.05 or lower was considered as statistical significant.

\section{RESULTS}

\section{In Silico pharmacokinetic studies}

This DS model predicts BBB penetration upon oral administration. Based on the quantitative linear regression model, (95\% confidence ellipse-blue line and 99\% confidence ellipsemagenta line) the results of the screened antioxidants are shown in the ADMET_PSA_2D versus ADMET_AlogP98 plane in Figure 1. The antioxidants were compared with the reference molecule diazepam. The relative results of $\mathrm{BBB}$ and ALogP98 values portrayed Naringenin as a potential molecule to cross BBB for showing its action as shown in Table 1 . None of the compounds exhibited any form of toxicity in the results obtained.

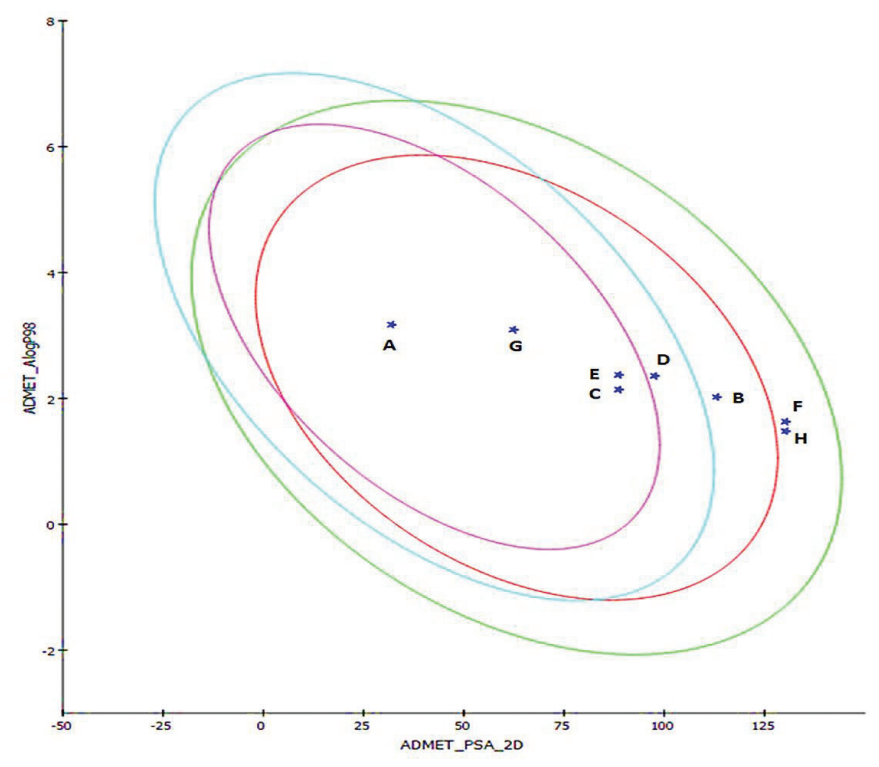

Figure 1. ADMET 2-D plot depicting the $95 \%$ and $99 \%$ confidence ellipses with compounds screened. 
Behavioral assessment in Drosophila melanogaster by negative geotaxis

ROT causes a change in negative geotaxis behavior in Drosophila. The results are clearly indicative of the locomotor deficit in flies when administered with ROT, as shown in Figure 2. The pretreatment with Naringenin, followed by induction of ROT, showed a better therapeutic effect.

\section{Biochemical parameters assessed from brain homogenates of Drosophila}

Upon ROT induction in the disease control, the level of LPO was significantly $(p<0.05)$ elevated and significant $(p<0.05)$ reductions in the levels of SOD, glutathione and CAT were observed compared to the control, which indicates the generation of reactive oxygen species (ROS). In the treatment group of ROT + Naringenin, there was a reduction in the LPO level and an increase in the levels of SOD, glutathione, and CAT. This significant $(p<0.05)$ response observed shows that naringenin has a potential effect on antioxidants. These results, as shown in Table 2, exhibit the potential to screen the molecule-selected candidate naringenin in the rodent model and to further convert it ito an SLN formulation.

\section{Evaluation of Naringenin loaded SLN}

Zeta potential is considered as the potential difference between the dispersion medium and the surface of a solid particle in a fluid. It is indicative of colloidal dispersion stability. The results so obtained, as shown in Figure 3, from the study show the zeta potential of $-11.9 \mathrm{mV}$. The maximum entrapment efficiency was $89.87 \% \pm 0.15 \%$ with the total drug content found to be

Table 1. In silico pharmacokinetic profiling for BBB penetration of select antioxidants using by DS

\begin{tabular}{|c|c|c|c|c|c|c|c|}
\hline Compounds & Solubility & Solubility Level & BBB & BBB Level & $\begin{array}{c}\text { Molecular Polar } \\
\text { Surface Area }\end{array}$ & AlogP98 & $\begin{array}{c}\text { ADMET PSA } \\
\text { 2D }\end{array}$ \\
\hline Diazepam (A) & -4.605 & 2 & 0.32 & 1 & 32.67 & 3.171 & 31.976 \\
\hline Epicatechin (B) & -2.445 & 3 & - & 4 & 110.38 & 2.021 & 113.007 \\
\hline Genistein (C) & -2.743 & 3 & -0.896 & 3 & 86.99 & 2.14 & 88.677 \\
\hline Hesperetin (D) & -3.157 & 3 & -0.97 & 3 & 96.22 & 2.357 & 97.607 \\
\hline Naringenin (E) & -2.942 & 3 & -0.824 & 3 & 86.99 & 2.373 & 88.677 \\
\hline Quercetin (F) & -2.633 & 3 & - & 4 & 127.45 & 1.63 & 130.308 \\
\hline Resveratrol (G) & -2.56 & 3 & -0.187 & 2 & 60.69 & 3.09 & 62.446 \\
\hline Taxifolin $(\mathrm{H})$ & -2.492 & 3 & - & 4 & 127.45 & 1.479 & 130.308 \\
\hline
\end{tabular}

The 2D mol file of molecules were downloaded from Pubchem, NCBI, USA.

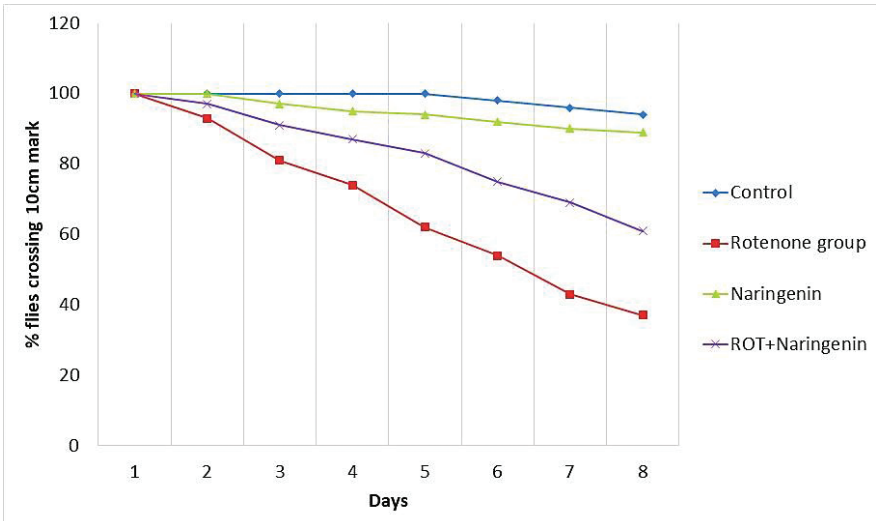

Figure 2. Negative geotaxis assay for behavioural assessment upon treatment in ROT-induced PD in Drosophila melanogaster $(n=50)$.

Table 2. Estimation of biochemical parameters of treated groups in Drosophila brain samples $(n=50)$.

\begin{tabular}{|c|c|c|c|c|}
\hline Groups & $\begin{array}{l}\text { LPO (nmol of malondialdehyde } \\
\text { /mg of protein) }\end{array}$ & $\begin{array}{c}\text { SOD (Units/minutes/mg of } \\
\text { protein) }\end{array}$ & $\begin{array}{c}\text { GSH (Units/mg of } \\
\text { protein) }\end{array}$ & $\begin{array}{c}\text { CAT (nmol/minutes } / \mathrm{mg} \text { of } \\
\text { protein) }\end{array}$ \\
\hline Control & $19.36 \pm 2.307$ & $2.427 \pm 0.288$ & $34.05 \pm 2.5$ & $0.1217 \pm 0.012$ \\
\hline ROT & $35.71 \pm 3.424$ & $1.097 \pm 0.267$ & $18.06 \pm 1.427$ & $0.0680 \pm 0.021$ \\
\hline $\begin{array}{l}\text { Naringenin (Pure } \\
\text { form) }\end{array}$ & $22.29 \pm 1.563$ & $2.798 \pm 0.348$ & $37.44 \pm 2350$ & $0.109 \pm 0.015$ \\
\hline ROT + Naringenin & $27.02 \pm 2.793$ & $1.802 \pm 0.255$ & $24.54 \pm 3.569$ & $0.089 \pm 0.015$ \\
\hline
\end{tabular}

All the numerical values are expressed as Mean \pm SE. Data was analyzed by One Way Analysis of Variance followed by Dunnet's post hoc test. 


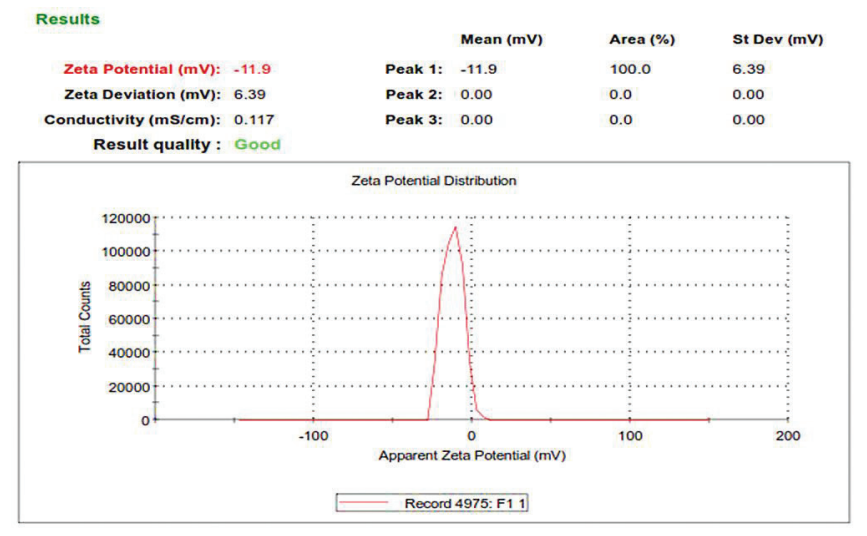

Figure 3. Zeta Potential Report of Naringenin Loaded SLNs.

Table 3. Drug entrapment efficiency and drug loading of Naringenin in SLNs using GMS

\begin{tabular}{cccc}
\hline Lipid & $\begin{array}{c}\text { Drug: lipid: surfactant } \\
\text { Ratio }\end{array}$ & $\begin{array}{c}\text { Entrapment efficiency } \\
(\%)\end{array}$ & $\begin{array}{c}\text { Drug loading } \\
(\%)\end{array}$ \\
\hline GMS & $1: 8: 4$ & 89.87 & 24.97 \\
\hline
\end{tabular}

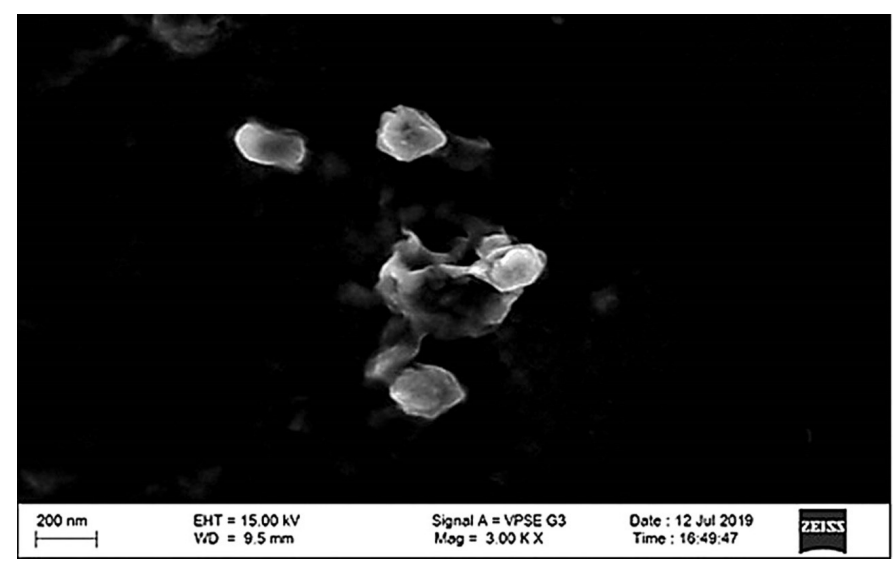

Figure 4. Morphology of Naringenin loaded SLNs by SEM analysis

$24.97 \pm 0.23 \mathrm{mg}$ of Naringenin, as summarized in Table 3. The morphological examination by SEM showed that nanoparticles were nearly spherical in shape majorly. The particle size of SLN, as shown in Figure 4, was found to be $134.5 \pm 20 \mathrm{~nm}$ and a PDI of 0.893 was observed.

\section{In vitro release studies and drug-release kinetics of Naringenin- loaded SLNs}

The release kinetic of Naringenin-loaded SLNs followed the Higuchi kinetic model as shown in Figure $5\left(R_{2}=0.9721\right)$.

Behavioral assessment in albino Wistar rats by muscle coordination and locomotor ability

Muscle coordinations assessed by the Rota rod apparatus were significantly deteriorated $(p<0.05)$ in the ROT group compared to the control as shown in Figure 6. When compared to the ROT group, L-Dopa group showed better significant retention time $(p<0.05)$ attributed to improvement in muscle coordination.

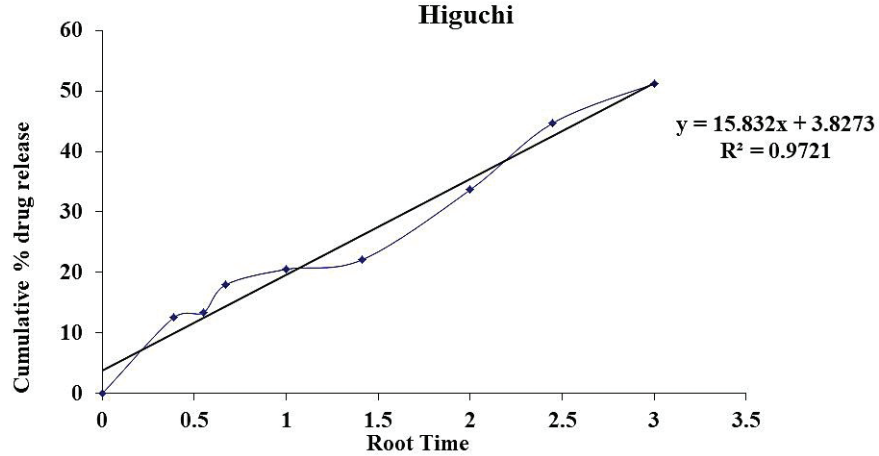

Figure 5. In vitro release profile of Naringenin loaded SLNs by dialysis technique in phosphate-buffered saline $(\mathrm{pH} 7.4)$ at $37^{\circ} \mathrm{C}$.

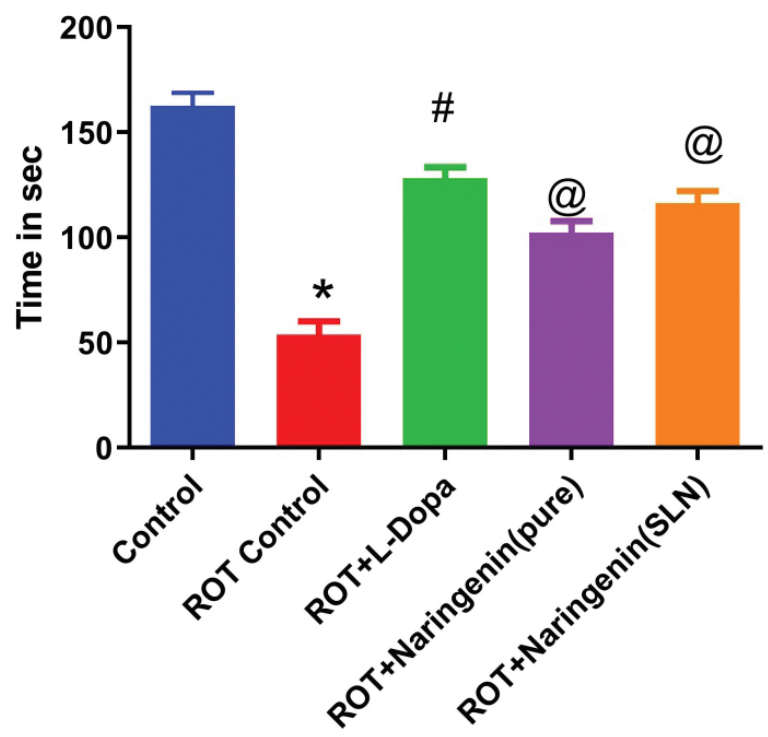

Figure 6. Rat muscle coordination activity using rotarod apparatus in various treatment groups in ROT-induced PD in rodent model $(n=6)$.

Both Naringenin treatment groups showed significant good muscle coordination when compared to ROT group. Similarly, the locomotor score evaluated by Acto-photometer was significantly diminished $(p<0.05)$ in the ROT group in comparison to the control group. This indicates the induction of oxidative stress and depletion in the neurons. Treatment with L-Dopa $(p<0.05)$ improved the locomotor score as compared to the diseased group as shown in Figure 7.

\section{Biochemical parameters assessed from brain homogenates of} rat

Upon ROT administration, there was a significant depletion $(p<0.05)$ in the total protein content in the brain compared to control. On L-Dopa treatment, a substantial increase $(p<0.05)$ in the protein level was observed than the ROT group. The amount of MDA was found to be significantly $(p<0.05)$ increased in the ROT group when compared to the control. The L-Dopa-treated groups showed a significant $(p<0.05)$ decrease in MDA level 
when compared to the ROT group. However, Naringenin in SLN formulation exhibited significant results compared to all treatment groups, as shown in Table 4.

Upon the administration of ROT, there was a decline $(p<0.05)$ in the SOD as compared to control, which indicates the extent of oxidative stress. L-Dopa treatment increased the SOD level in the rat brain when compared to the ROT group. The naringenin pure form and SLN formulation showed a remarkable increase in the SOD in the rat brain. It was revealed that there was a reduction $(p<0.05)$ in the GSH level after the administration of ROT. When treated with L-Dopa, there was an increase in thew GSH level, followed by the naringenin pure form and SLN group as compared to disease control. The level of CAT in ROT-infused rats has decreased $(p<0.05)$ as compared to the control group as shown in the figure. The elevated $(p<0.05)$ level of CAT was observed in Naringenin pure form and SLN-treated groups. The treatment with the Naringenin had showed marked reduction as compared to the standard L-Dopa group. The results exhibit that treatment with Naringenin in SLN formulation also show similar results as that of L-Dopa group. Treatment with Naringenin also shows that the results are comparable to Naringenin-SLN formulation.

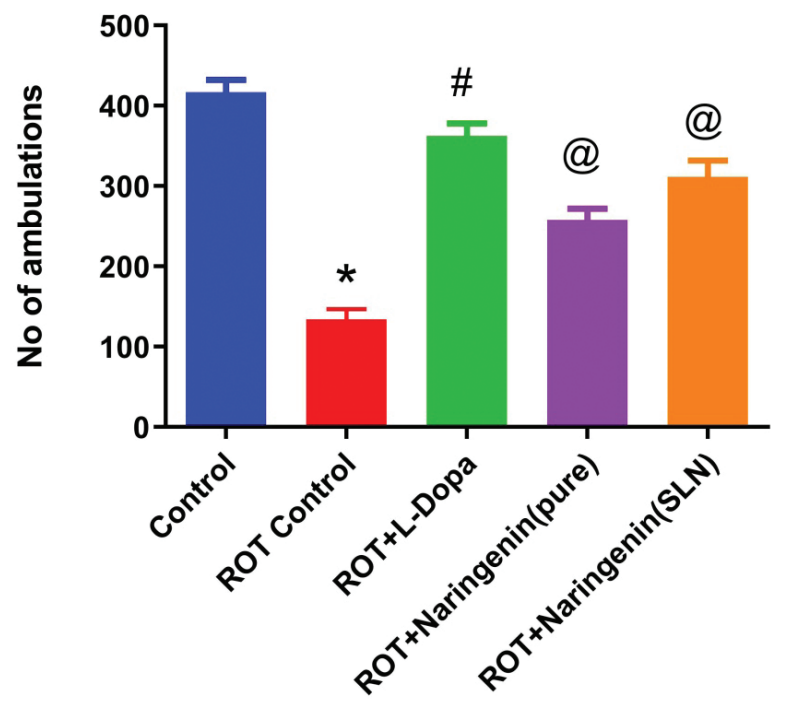

Figure 7. Locomotor activity using digital actophotometer in various treatment groups in ROT-induced PD in the rodent model $(n=6)$.

\section{Histopathological examinations}

The histopathological section of the brain samples were focused on the substantia nigra region via Motic microscope, USA. Control growth shows normal architecture of brain neuron as shown in Figure 8. There are intact neuroglial cells with no protein aggregates indicates absence of PD. However, neurodegeneration with accumulation of protein aggregation was seen in the disease control group. Our treatment shows the protection of neuronal damage. The section of SLN shows higher amount of protection measured by the lack/no damage to neurons. This study revealed the presence of Lewy bodies and protein aggregation in the ROTinduced disease group. The Lewy body formation is reduced by pretreatment with Naringenin. All the results so obtained are discussed further.

\section{DISCUSSION}

The current treatment strategies employed to manage the PD ranges from dopamine agonists, monoamine oxidase inhibitors to anti-cholinergic drugs. The aim of the treatment available was to mainly increase or substitute dopamine levels in the brain to balance the effect of acetyl choline. Both these neurotransmitters have to work in tandem and balance. However, as the extent of degeneration increases, no drug classes have the ability to halt the disease progression and protect the existing neurons. In the current global scenario of experimental and clinical research, gene therapy, PD vaccine, stem cell therapy, and neuronal cell transplantation are few ongoing innovations to treat PD. However, such research is still on the clinical phase and did not proceed further for marketing. In addition, currently available treatment does not target the disease pathogenesis, as they mainly focus on

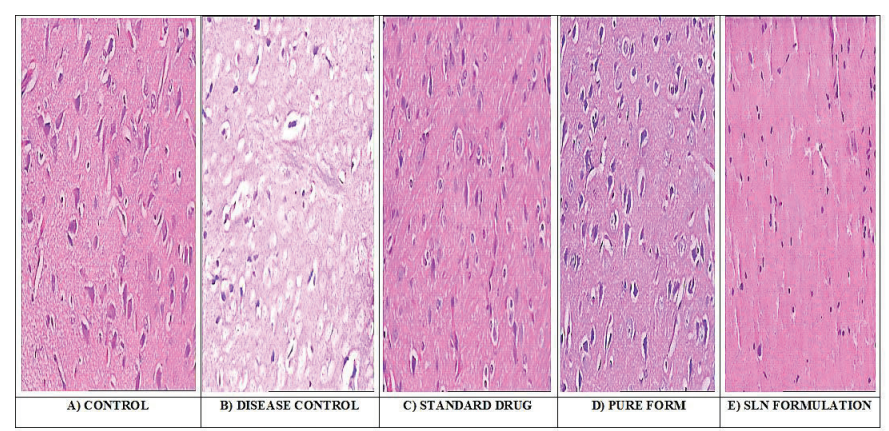

Figure 8. Images showing the sections of substantia nigra of treated groups in the rodent model by $\mathrm{H} / \mathrm{E}$ staining $(n=6)$

Table 4. Estimation of biochemical parameters of treated groups of isolated rat brain samples $(n=6)$.

\begin{tabular}{|c|c|c|c|c|c|}
\hline Groups & $\begin{array}{c}\text { Concentration of } \\
\text { protein (mg/100 } \mathrm{mg} \\
\text { of tissue) }\end{array}$ & $\begin{array}{c}\text { LPO (nmol of MDA/mg } \\
\text { of protein) }\end{array}$ & $\begin{array}{c}\text { SOD (Units/minutes } / \mathrm{mg} \\
\text { of protein) }\end{array}$ & $\begin{array}{c}\text { GSH (Units/mg of } \\
\text { protein) }\end{array}$ & $\begin{array}{c}\text { CAT (nmol/ } \\
\text { minutes/mg of } \\
\text { protein) }\end{array}$ \\
\hline Control & $41.53 \pm 0.306$ & $9.138 \pm 1.062$ & $16.39 \pm 1.810$ & $18.14 \pm 2.562$ & $9.49 \pm 1.590$ \\
\hline ROT Control & $20.33 \pm 4.166$ & $18.34 \pm 1.054$ & $5.54 \pm 1.842$ & $5.830 \pm 1.696$ & $3.65 \pm 1.23$ \\
\hline ROT + L-Dopa & $34.36 \pm 3.549$ & $13.27 \pm 1.792$ & $13.54 \pm 1.971$ & $15.07 \pm 0.107$ & $8.59 \pm 1.38$ \\
\hline ROT + Naringenin & $24.89 \pm 2.263$ & $15.05 \pm 0.975$ & $9.37 \pm 2.315$ & $11.24 \pm 1.356$ & $5.37 \pm 1.09$ \\
\hline $\begin{array}{l}\text { ROT + Naringenin- } \\
\text { SLN }\end{array}$ & $28.73 \pm 1.059$ & $11.35 \pm 1.616$ & $12.62 \pm 2.541$ & $13.97 \pm 2.260$ & $6.95 \pm 0.65$ \\
\hline
\end{tabular}

All the numerical values are expressed as Mean \pm SE. Data was analyzed by One Way Analysis of Variance followed by Dunnet's post hoc test 
the management towards symptomatic relief for a better quality of life (Jankovic \& Poewe, 2012; Tsouli \& Konitsiotis, 2010). Our study mainly concentrated on the neuroprotective potential of natural polyphenol compounds to suppress the disease progression. This is achieved by reducing the damage to neurons and delay progression of disease (Solanki et al., 2015).

As the age progress, the endogenous antioxidant defense system deteriorates, whereas the production of ROS gradually increases. This is possible when the body is exposed to harmful neurotoxins on a chronic basis. In this study, we chose one such agent - ROT - which is a pesticide that can produce neurodegeneration. ROT is reported to act by disrupting the mitochondrial system, initiate protein aggregation and ultimately cause DNA damage. In the multifactorial pathogenesis of PD, reports state that oxidative stress activates a malicious process of LPO formation, followed by increased $\alpha$-synuclein aggregates, consequently transforming into Lewy bodies. These are often associated with neuronal dysfunction that triggers the onset of PD symptoms (Magalingam et al., 2015).

Hence, oxidative stress increasing with age is one such known cause for neuron degradation. Of which, ROS formed in the brain contributes to the imbalance of the neuron architecture in the brain. To treat such anomalies, antioxidants have been studied extensively and reported to provide beneficial effects to counteract against the ROS (Pohl \& Kong Thoo Lin, 2018). With regard to today's research perspective, the possible mechanisms by which how an antioxidant can prevent neurodegenerative conditions is well established. This includes some major targets of Sirtuin Pathway, NF-кB pathway, and Nrf-Z/ARE pathway (Vauzour, 2012). But failure in ADME or toxicity is a major cause for the failure in efficacy and selectivity of drugs, in this case the antioxidants (Youdim et al., 2004). Therefore, an in silico pharmacokinetics modeling software - DS - was employed to virtually understand and detect the ADME parameters of the antioxidants in comparison with a drug like Diazepam. The algorithmic model by Cheng et al. (2002) helped to predict pharmacokinetic parameters of antioxidants. The ellipses intervals of $95 \%-99 \%$ described the nature of the molecule studied in this model (Egan et al., 2000). Resveratrol exhibited best results, yet the pure form of resveratrol is very expensive. Naringenin, hesperetin, and genistein also showed comparable good solubility and exhibited better results in BBB penetration in the ADMET PSA_2D versus ADMET_AlogP98 plane.

Naringenin is a natural flavonoid compound that has been reported to be an efficient anti-oxidant, scavenge free radicals, and possess anti-inflammatory action (Devan \& Janardhanam, 2011). The organ protective effect of Naringenin has been explored on various diseases, such as ischemic stroke, cerebral ischemia, ischemia reperfusion cerebral injury, ischemic heart disease adipolylic activity,_and even anti-cancer activity (Figueira et al., 2017; Habtemariam, 2019). A study based on availability of Naringenin in brain on intravenous administration gave us a perception on its nature to cross BBB (Fu et al., 2015; Peng et al., 1998). With these data and in silico results obtained, it was imperative to choose Naringenin due to its cost effectiveness, good physicochemical properties, and sufficient level to cross BBB to exert the pharmacological action (Ponnan et al., 2013).

SLN are one of efficient drug delivery systems that can offer extended and constant drug release. In addition, they also offer improved stability, involve nontoxic nanocarrier systems, and increase the bioavailability of poor absorbing drugs upon administration. A study conducted by Wu et al. (2016) has shown that GMS was selected to prepare SLNs with sufficient pharmacokinetic parameters optimized for a pulmonary administration. Similarly, (Lou et al., 2014) also have conducted an in vitro study on naringenin loaded nanoparticles for better neuroprotective ability and antioxidant effect against 6-OHDAinduced neurotoxicity in SH-SY5Y cells (Md et al., 2019). In a study conducted by Bayón-Cordero et al. (2019) SLNs offered improve drug bioavailability than the conventional administration free drug. In addition, they would also act as carriers that can interact with biological barriers and offer better permeation across such barriers. The formulation would also offer stable formulation, increased drug bioavailability and better permeability across BBB. With the scientific evidences and in silico studies carried out, Naringenin in SLNs formulation was hypothesized to evaluate the neuroprotective potential on PD in two experimental animals Drosophila melanogaster and Wistar rats by ROT-induced PD.

In recent times, Drosophila melanogaster or fruit fly has been used as an alternative model to study the neuro-modulatory properties of the natural polyphenolic molecules (Manjunath \& Muralidhara, 2015). The genetic tool of Drosophila is well established it is found that there is striking similarities with the human genome especially brain architecture. Due to their short life span and sophisticated central nervous system with BBB, it is one of best alternative model can used for the neuroprotective evaluation of PD. However, due to its small morphology, it is difficult to evaluate parameters such as enhanced bioavailability by advanced drug delivery systems, histopathological analysis in depth. Hence, for this reason Wistar albino rats were also used to evaluate the neuroprotective action.

Results of the present study confirm the oxidative injury caused by ROT in neurons as evident from the data so obtained. ROT administered in Drosophila has led to the neuronal degeneration which is confirmed by assessing the behavioral parameters. Negative geotaxis is a distinctive behavioral trait of the flies, where in they start to ascend the wall of a cylinder upon a stimulus. This is one such behavior that senesces in Drosophila and is always indicative of disease induction. In PD induced flies, they lose this ability and tend remains at the bottom (Kujawska \& Jodynis-Liebert, 2018). In addition, there is increase in oxidative stress marker of LPO in tissue homogenate of the brain samples which points to the confirmation of ROT-induced oxidative damage in neurons. The loss of negative geotaxis behavior in flies confirms the PD induction.

Humans are equipped with antioxidant defense systems including glutathione (GSH), dietary antioxidants such as ascorbic acid, tocopherol, proteinous metal chelating agents and antioxidant enzyme system(Habtemariam, 2019). One such abundantly consumed antioxidant is Naringenin. It is abundantly present in citrus fruits, berries, tomatoes and so on. A study by Kumar et al. (2010) has shown that naringenin elevated the SOD and glutathione peroxidase levels upon treatment. Hence, by a preliminary study of naringenin treatment conducted in Drosophila model was conclusive of neuroprotective potential. In this study, the treatment with Naringenin when given alone in flies has reduced the level of oxidative stress and increase in natural anti-oxidant present in its system (de Andrade Teles et al., 2018; Venkatachalam et al., 2013) However, it was still unclear whether this concentration would be enough to prevent neuronal damage by oxidative stress in PD. Henceforth, to increase bioavailability of Naringenin; it was converted into SLN formulation (Gastaldi 
et al., 2014). This type of advanced drug delivery system has been proven to increase the availability of drug content in brain for sustained activity.

A study conducted by Raza et al. (2013) showed that naringenin up regulated the antioxidant system in the treated animals. In addition to this, it is reported to inhibit the expression of $N F-\kappa B$ and activation of genes that can trigger the inflammation cascade. The mechanism established in an inflammatory condition is applicable to ROT-induced PD. Similarly, a study report state that naringenin pretreatment produces decreased expression of $N F-\kappa B$ protein complexes, inducible nitric oxide synthase, and cyclooxygenase-2 genes were found in an experimental model of focal cerebral ischemia/reperfusion induced inflammation. With this background, the neuroprotective activity of Naringenin was evaluated in the rodent model in both case scenarios that is: in pure form of Naringenin and SLN formulation of Naringenin. Results of the biochemical parameters such as SOD, GSH, and CAT in the brain are indicative of a beneficial innate antioxidant system. Subsequently, the oxidative marker such as LPO was estimated to clearly conclude that the ROS formed by ROT are negated by the treatment of Naringenin. This is comparable to results of the standard drug treatment given by standard drug available in market Levodopa. This is clear indicative of the potential of naringenin to shows its neuroprotective action across BBB. With results so obtained and scientific evidences reviewed, Naringenin in SLNs has exhibited promising neuroprotective potential in studies conducted on Drosophila melanogaster and Wistar rats by ROTinduced PD model.

\section{CONCLUSION}

Treatment of PD may be simple yet it is intricate in choosing the correct strategy. The need of this therapeutic direction was to prevent the neuronal damage occurring in patients and reduce the disease progression in PD. Oxidative stress is one of the major causes for pathogenesis leading to PD. Free radicals generated in the body react with membrane lipids leading to LPO, membrane injury, and nerve cell death. This can be achieved by supplementing the standard drug therapy with antioxidants. Naringenin is one such potential antioxidant and was studied for its neuroprotective potential. In this study, naringenin in SLNs exerts good neuroprotective effect on PD. However, the pharmacological assessment was limited to neuroprotective potential of naringenin in SLN formulation. However, it may be planned and designed to assess the better therapeutic efficacy in combination with Levodopa as an adjuvant drug in the future studies.

\section{ACKNOWLEDGMENTS}

The authors would like to acknowledge and thank the Department of Science and Technology - Fund for Improvement of Science and Technology Infrastructure in Universities and Higher Educational Institutions (DST-FIST), Govt. of India, New Delhi, for their infrastructure and support to our department. The authors wholeheartedly thank the Heads of Dept. of Pharmacology, Dept. of Pharmaceutics, Dept. of Pharmaceutical analysis, and Dept. of Pharm. Biotechnology for providing the opportunity to bring out this research work. The authors thank Dr. S. P. Dhanabal, the Principal, JSS College of Pharmacy, Ooty, for providing all the needed support and encouragement to do this work.

\section{AUTHOR CONTRIBUTIONS}

All authors made substantial contributions to conception and design, acquisition of data, or analysis and interpretation of data; took part in drafting the article or revising it critically for important intellectual content; agreed to submit to the current journal; gave final approval of the version to be published; and agree to be accountable for all aspects of the work.

\section{CONFLICTS OF INTEREST}

The authors report no conflicts of interest in this work.

\section{ETHICAL APPROVAL}

This study does not involve the use of animals or human subjects.

\section{FUNDING}

This work is partially supported by the Tamil Nadu State Pharmaceutical Welfare Trust, Tamilnadu State, India, which is a registered trust whose sole objective is to screen and identify scientific proposals for awards. Our research work was awarded at the state level and financially supported for purchase of chemicals

\section{REFERENCES}

Apostolova N, Victor VM. Molecular strategies for targeting antioxidants to mitochondria: therapeutic implications. Antioxid Redox Signal, 2015; 22(8):686-729.

Bayón-Cordero L, Alkorta I, Arana L. Application of solid lipid nanoparticles to improve the efficiency of anticancer drugs. Nanomaterials, 2019; 9(3):474.

Bilia AR, Isacchi B, Righeschi C, Guccione C, Bergonzi MC. Flavonoids loaded in Nanocarriers: an opportunity to increase oral bioavailability and bioefficacy. Food Sci Nutr, 2014; 05(13):1212-327.

Campos FL, Carvalho MM, Cristovão AC, Je G, Baltazar G, Salgado AJ, Kim, YS, Sousa N. Rodent models of Parkinson's disease: beyond the motor symptomatology. Front Behav Neurosci, 2013; 7:175.

Chen C, Wei YZ, He XM, Li DD, Wang GQ, Li JJ, Zhang F. Naringenin produces Neuroprotection against LPS-induced dopamine neurotoxicity via the inhibition of microglial NLRP3 inflammasome activation. Front Immunol, 2019; 10:936.

Cheng A, Diller DJ, Dixon SL, Egan WJ, Lauri G, Merz KM. Computation of the physio-chemical properties and data mining of large molecular collections. J Comput Chem, 2002; 23(1):172-83.

Collins MA. Ethanol and adult CNS neurodamage: oxidative stress, but possibly not excitotoxicity. Front Biosci, 2012; E4(1):1358.

Cui Q, Wang L, Wei Z, Qu W. Continual Naringin treatment benefits the recovery of traumatic brain injury in rats through reducing oxidative and inflammatory alterations. Neurochem Res, 2014; 39(7):1254-62.

de Andrade Teles RB, Diniz TC, Costa Pinto TC, de Oliveira Júnior RG. Gama e Silva M, de Lavor ÉM, Fernandes AWC, de Oliveira AP, de Almeida Ribeiro FPR, da Silva AAM, Cavalcante TCF, Quintans Júnior LJ, da Silva Almeida JRG. Flavonoids as therapeutic agents in Alzheimer's and Parkinson's Diseases: A Systematic Review of Preclinical Evidences. Oxi Med Cell Longev, 2018; 2018:1-21.

Devan S, Janardhanam VA. Effect of Naringenin on metabolic markers, lipid profile and expression of GFAP in C6 glioma cells implanted rat's brain. Ann Neurosci, 2011; 18(4):151-5.

Dorsey ER, Elbaz A, Nichols E, Abd-Allah F, Abdelalim A, Adsuar JC, Ansha MG, Brayne C, Choi JYJ, Collado-Mateo D, Dahodwala N, Do HP, Edessa D, Endres M, Fereshtehnejad SM, Foreman KJ, Gankpe FG, Gupta R, Hankey GJ, Murray CJL. Global, regional, and national burden of Parkinson's disease, 1990-2016: a systematic analysis for the global burden of disease study 2016. Lancet Neurol, 2018; 17(11):939-53.

Egan WJ, Merz KM, Baldwin, JJ. Prediction of drug absorption using multivariate statistics. J Med Chem, 2000; 43(21):3867-77.

Ellman GL. Tissue sulfhydryl groups. Arch Biochem Biophys, 1959; 82(1):70-7.

Figueira I, Garcia G, Pimpão RC, Terrasso AP, Costa I, Almeida AF, Tavares L, Pais TF, Pinto P, Ventura MR, Filipe A, McDougall GJ Stewart D, Kim KS, Palmela I, Brites D, Brito MA, Brito C, Santos 
CN. Polyphenols journey through blood-brain barrier towards neuronal protection. Sci Rep, 2017; 7(1):11456.

Fu W, Zhuang W, Zhou S, Wang X. Plant-derived neuroprotective agents in Parkinson's disease. Am J Transl Res, 2015; 7(7):1189-202.

Gastaldi L, Battaglia L, Peira E, Chirio D, Muntoni E, Solazzi I, Gallarate M, Dosio F. Solid lipid nanoparticles as vehicles of drugs to the brain: current state of the art. Eur J Pharm Biopharm, 2014; 87(3):433-44.

Goetz CG. The history of Parkinson 's disease: early clinical descriptions and neurological therapies. Cold Spring Harb Perspect Med, 2011; 1(1):a008862.

Habtemariam S. The Nrf2/HO-1 axis as targets for flavanones: neuroprotection by pinocembrin, Naringenin, and eriodictyol. Oxid Med Cell Longev, 2019; 2019 1-15.

Hauser RA. Levodopa: past, present, and future. Eur Neurol, 2009; 62(1):1-8

Hwang O. Role of oxidative stress in Parkinson's disease. Exp Neurobiol, 2013; 22(1):11.

Jankovic J, Poewe W. Therapies in Parkinson's disease. Curr Opin Neurol, 2012; 25(4):433-47.

Joseph JA, Shukitt-Hale B, Denisova NA, Bielinski D, Martin A, McEwen JJ, Bickford PC. Reversals of age-related declines in neuronal signal transduction, cognitive, and motor behavioral deficits with Blueberry, Spinach, or strawberry dietary supplementation. J Neurosci, 1999; 19(18):8114-21

Kaur IP, Bhandari R, Bhandari S, Kakkar V. Potential of solid lipid nanoparticles in brain targeting. J Control Release, 2008; 127(2):97-109.

Kim S, Chen J, Cheng T, Gindulyte A, He J, He S, Li Q, Shoemaker BA, Thiessen PA, Yu B, Zaslavsky L, Zhang J, Bolton EE. PubChem 2019 update: improved access to chemical data. Nucleic Acids Res, 2019; 47(D1):D1102-9.

Kujawska M, Jodynis-Liebert J. Polyphenols in Parkinson's disease: a systematic review of in vivo studies. Nutrients, 2018; 10(5):642.

Kumar A, Prakash A, Dogra S. Naringin alleviates cognitive impairment, mitochondrial dysfunction and oxidative stress induced by d-galactose in mice. Food Chem Toxicol, 2010; 48(2):626-32.

Lou H, Jing X, Wei X, Shi H, Ren D, Zhang X. Naringenin protects against 6-OHDA-induced neurotoxicity via activation of the $\mathrm{Nrf2}$ / ARE signaling pathway. Neuropharmacology, 2014; 79:380-8.

Magalingam KB, Radhakrishnan AK, Haleagrahara N. Protective mechanisms of flavonoids in Parkinson's disease. Oxid Med Cell Longev, 2015: 2015:1-14.

Manjunath MJ, Muralidhara. Standardized extract of Withania somnifera (Ashwagandha) markedly offsets rotenone-induced locomotor deficits, oxidative impairments and neurotoxicity in Drosophila melanogaster. J Food Sci Technol, 2015; 52(4):1971-81

Md S, Alhakamy NA, Aldawsari HM, Asfour HZ. Neuroprotective and antioxidant effect of Naringenin-loaded nanoparticles for nose-to-brain delivery. Brain Sci, 2019; 9(10):275.

Mukherjee P, Ahamed K, Kumar V, Mukherjee K, Houghton P. Protective effect of biflavones from Araucaria bidwillii hook in rat cerebral ischemia/reperfusion induced oxidative stress. Behav Brain Res, 2007; 178(2):221-8

Ohkawa H, Ohishi N, Yagi K. Assay for lipid peroxides in animal tissues by thiobarbituric acid reaction. Anal Biochem, 1979; 95(2):351-8.

Peng HW, Cheng FC, Huang YT, Chen CF, Tsai TH. Determination of Naringenin and its glucuronide conjugate in rat plasma and brain tissue by high-performance liquid chromatography. J Chromatogr B Biomed Sci Appl, 1998; 714(2):369-74.

Pohl F, Kong Thoo Lin P. The potential use of plant natural products and plant extracts with antioxidant properties for the prevention/ treatment of neurodegenerative diseases: in vitro, in vivo and clinical trials. Molecules, 2018; 23(12):3283.

Ponnan P, Gupta S, Chopra M, Tandon R, Baghel AS, Gupta G, Prasad AK, Rastogi RC, Bose M, Raj HG. 2D-QSAR, docking studies, and in silico ADMET prediction of polyphenolic acetates as substrates for protein acetyltransferase function of glutamine synthetase of mycobacterium tuberculosis. ISRN Struct Biol, 2013; 2013:1-12.
Raza SS, Khan MM, Ahmad A, Ashafaq M, Islam F, Wagner AP, Safhi MM, Islam F. Neuroprotective effect of Naringenin is mediated through suppression of NF- $\mathrm{KB}$ signaling pathway in experimental stroke. Neuroscience, 2013; 230:157-71.

Saggu H, Cooksey J, Dexter D, Wells FR, Lees A, Jenner P, Marsden CD. A selective increase in particulate superoxide dismutase activity in Parkinsonian substantia Nigra. J Neurochem, 1989; 53(3):692-7.

Sita G, Hrelia P, Tarozzi A, Morroni F. Isothiocyanates are promising compounds against oxidative stress, neuroinflammation and cell death that may benefit neurodegeneration in Parkinson's disease. Int J Mol Sci, 2016; 17(9):1454.

Solanki I, Parihar P, Mansuri ML, Parihar MS. Flavonoid-based therapies in the early management of neurodegenerative diseases. Adv Nutr, 2015; 6(1):64-72

Sun AY, Wang Q, Simonyi A, Sun GY. Botanical phenolics and brain health. Neuromolecular Med, 2008; 10(4):259-74.

Tsouli S, Konitsiotis S. How should we treat a patient with early Parkinson's disease? Treatment initiation in Parkinson's disease. Int J Clin Pract, 2010; 64(9):1210-9.

Vauzour D. Dietary polyphenols as modulators of brain functions: biological actions and molecular mechanisms underpinning their beneficial effects. Oxid Med Cell Longev, 2012:2012:1-16.

Venkatachalam S, Muthaiah VP, Venkitasamy L, Michael F, Chandrasekar K. Neuroprotective role of Naringenin on carbaryl induced neurotoxicity in mouse neuroblastoma cells. J Pharmacol Pharmacother, 2013; 4(3):192

Vos T, Barber RM, Bell B, Bertozzi-Villa A, Biryukov S, Bolliger I, Charlson F, Davis A, Degenhardt L, Dicker D, Duan L, Erskine H, Feigin VL, Ferrari AJ, Fitzmaurice C, Fleming T, Graetz N, Guinovart C, Haagsma J, Murray CJ. Global, regional, and national incidence, prevalence, and years lived with disability for 301 acute and chronic diseases and injuries in 188 countries, 1990-2013: a systematic analysis for the Global Burden of Disease Study 2013. Lancet, 2015; 386(9995):743-800.

WHO. Neurological disorders: public health challenges. Available via https://www.who.int/mental_health/neurology/neurodiso/en/ (Accessed 08 October 2020).

Wokes F, Still BM. The estimation of protein by the biuret and Greenberg methods. Biochem J, 1942; 36(10-12):797-806.

Wu C, Ji P, Yu T, Liu Y, Jiang J, Xu J, Zhao Y, Hao Y, Qiu Y, Zhao W. Naringenin-loaded solid lipid nanoparticles: preparation, controlled delivery, cellular uptake, and pulmonary pharmacokinetics. Drug Des Devel Ther, 2016; 10:911-25.

Xiong N, Huang J, Zhang Z, Zhang Z, Xiong J, Liu X, Jia M, Wang F, Chen C, Cao X, Liang Z, Sun S, Lin Z, Wang T. Stereotaxical infusion of Rotenone: a reliable rodent model for Parkinson's disease. PLoS One, 2009; 4(11):e7878.

Youdim KA, Qaiser MZ, Begley DJ, Rice-Evans CA, Abbott, NJ. Flavonoid permeability across an in situ model of the blood-brain barrier. Free Radic Biol Med, 2004; 36(5):592-604.

Zafar KS, Siddiqui A, Sayeed I, Ahmad M, Salim $\mathrm{S}$, Islam F. Dose-dependent protective effect of selenium in rat model of Parkinson's disease: neurobehavioral and neurochemical evidences: effect of selenium in rat model of Parkinson's disease. J Neurochem, 2003; 84(3):438-46.

\section{How to cite this article:}

Mani M, Balasubramanian S, Manikandan KR, Kulandaivel B. Neuroprotective potential of Naringenin loaded solid-lipid nanoparticles against Rotenone-induced Parkinson's disease model. J Appl Pharm Sci, 2021; 11(02):019-028. 


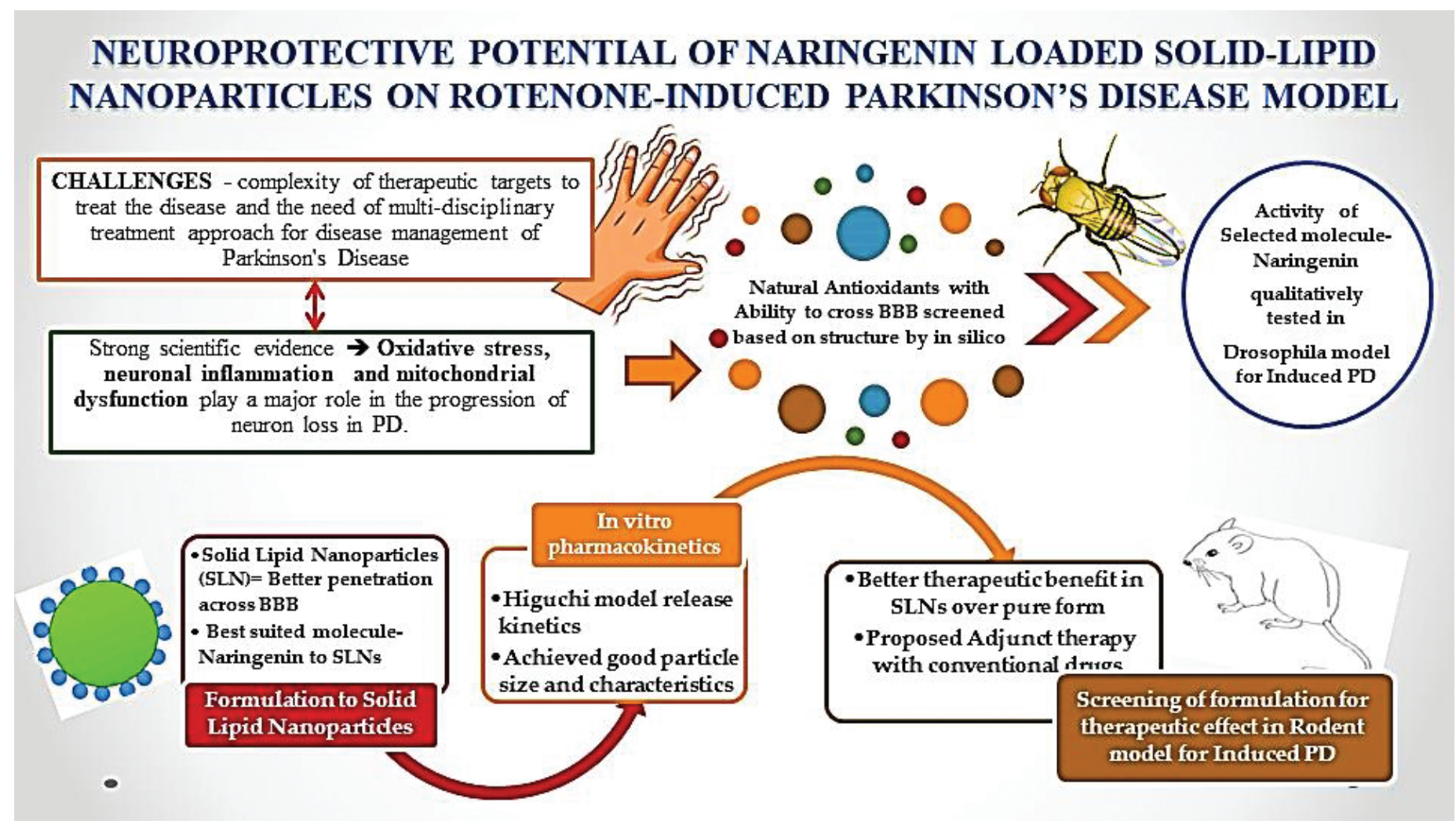

